\title{
Inhibition of lymphangiogenic factor VEGF-C expression and production by the histone deacetylase inhibitor suberoylanilide hydroxamic acid in breast cancer cells
}

\author{
HSUEH-TSEN CHENG ${ }^{1}$ and WEN-CHUN HUNG ${ }^{2}$ \\ ${ }^{1}$ Institute of Biomedical Sciences, National Sun Yat-Sen University, Kaohsiung 804; \\ ${ }^{2}$ National Institute of Cancer Research, National Health Research Institutes, Tainan 704, Taiwan, R.O.C.
}

Received September 7, 2012; Accepted November 26, 2012

DOI: $10.3892 /$ or.2012.2188

\begin{abstract}
Suberoylanilide hydroxamic acid (SAHA), a potent histone deacetylase (HDAC) inhibitor, has been shown to exert anticancer effects in various types of human cancer and is now used in the clinic for cancer treatment. In addition to cytostatic and cytotoxic activities, SAHA also represses angiogenesis to inhibit tumor growth. However, the effect of SAHA on tumor lymphangiogenesis, a step in which cancer cells produce prolymphangiogenic factors such as vascular endothelial growth factor-C (VEGF-C) to stimulate proliferation and migration of lymphatic endothelial cells, remains largely unclear. In this study, we investigated the expression of VEGF-C in breast cancer cell lines and found that VEGF-C was highly expressed in MDA-MB-231, MCF-7, MDA-MB-453 and BT-474 cells. SAHA inhibited VEGF-C expression in a dose-dependent manner in these cell lines. The secretion of VEGF-C into conditioned medium was also suppressed. We cloned human VEGF-C gene promoter and demonstrated that SAHA directly repressed promoter activity in MDA-MB-231 cells. Promoter deletion assay suggested that SAHA repressed VEGF-C via the $-185 /+38$ region which contained several transcription factor binding sites. Notably, we found that SAHA reduced $\mathrm{Sp} 1$, but not Sp3 and NF- $\mathrm{B}$ protein levels. Treatment with Sp1 inhibitor mithramycin A also inhibited VEGF-C expression in breast cancer cells. In addition, enforced expression of Sp1 partially rescued the inhibition of VEGF-C by SAHA. Collectively, our results suggest that SAHA inhibits VEGF-C expression in breast cancer cells via transcriptional repression and this drug may exert anti-lymphangiogenic activity in cancer treatment.
\end{abstract}

Correspondence to: Professor Wen-Chun Hung, National Institute of Cancer Research, National Health Research Institutes, 367 Shengli Road, Tainan 704, Taiwan, R.O.C.

E-mail: hung1228@ms10.hinet.net

Key words: histone deacetylase, suberoylanilide hydroxamic acid, $\mathrm{Sp1}$, vascular endothelial growth factor-C

\section{Introduction}

Epigenetic alteration is a general phenomenon during carcinogenesis (1). Both DNA methylation and histone modification play critical roles in the control of expression of oncogenes and tumor suppressor genes $(2,3)$. One of the most important histone modifications is acetylation which is controlled by histone acetyltransferases (HATs) and histone deacetylases (HDACs). These enzymes are considered to be crucial targets for the development of anticancer drugs. Previous studies demonstrated that HDAC inhibitors show potent anticancer activities against different types of human cancer (4-6).

HDAC inhibitors are classified into different groups based on their chemical structures and their ability to inhibit individual HDACs $(7,8)$. The first class is short chain fatty acids including butyrate and valproate. The second class is hydroxamic acid derivatives such as trichostatin A, suberoylanilide hydroxamic acid (SAHA, also known as vorinostat). The third class is benzamide and the fourth class is cyclic tetrapeptide. Among them, SAHA is one of the pioneer HDAC inhibitors that has entered clinical therapy and is now approved for the treatment of cutaneous $\mathrm{T}$ cell lymphoma $(9,10)$. This drug is also undergoing clinical trials for the treatment of solid tumors, including non-small cell lung cancer and breast cancer. The anticancer effects of SAHA are mediated by different mechanisms. First, SAHA directly causes apoptosis in cancer cells $(11,12)$. Second, SAHA upregulates anti-proliferative genes, such as p21, to inhibits proliferation $(13,14)$. Third, SAHA acts as an immune modulating drug to improve anticancer immune surveillance $(15,16)$. Fourth, SAHA suppresses angiogenesis to attenuates tumor growth in vivo (17).

Lymphangiogenesis is the process by which cancer cells promote the proliferation of lymphatic endothelial cells and enhance the migration of these cells toward tumor part (18). Previous studies demonstrated that induction of lymphangiogenesis is strongly associated with tumor metastasis and poor prognosis in cancer patients (19-21). The key step in inducing lymphangiogenesis is the production of lymphangiogenic factors by cancer cells. Among the lymphangiogenic factors studied, vascular endothelial growth factor-C (VEGF-C) has received considerable attention since lymphatic endothelial 
cells express high levels of its cognate receptor VEGFR3 and their proliferation is potently stimulated by VEGF-C.

Although SAHA was able to inhibit angiogenesis in different types of cancer, its effect on lymphangiogenesis has not been demonstrated. In this study, we investigated the expression of VEGF-C in breast cancer cell lines and its regulation by SAHA. In addition, we tried to elucidate the underlying mechanism by which SAHA modulated VEGF-C transcription.

\section{Materials and methods}

Cell culture. Human breast cancer cell lines MDA-MB-231, MCF-7, MDA-MB-453 and the normal breast cell line M10 were purchased from the cell bank of the National Health Research Institute (Maoli, Taiwan). MDA-MB-231 and MDA-MB-453 cells were grown in the L15 medium (Invitrogen, Carlsbad, CA, USA) containing 10\% FCS, L-glutamate and antibiotics. MCF-7 and M10 cells were cultured in the MEM medium (Invitrogen) containing 10\% FCS and antibiotics. BT-474 breast cancer cells were kindly provided by Dr Jin-Yuh Shew (National Taiwan University, Taiwan) and were maintained in the DMEM/F12 medium (Invitrogen) supplemented with $10 \%$ FCS, L-glutamate, nonessential amino acids, sodium pyruvate and antibiotics. All cells were cultured at $37^{\circ} \mathrm{C}$ in a humanized incubator with $5 \% \mathrm{CO}_{2}$.

Reagents and antibodies. SAHA was purchased from LC Laboratories (Woburn, MA, USA). SAHA was dissolved in DMSO at a concentration of $20 \mathrm{mM}$ and stored at $-70^{\circ} \mathrm{C}$. It was diluted in the culture medium to different working concentrations and used for cell treatment. Mithramycin A was purchased from Sigma-Aldrich (St. Louis, MO, USA). Antibody against VEGF-C was purchased from R\&D Systems (Minneapolis, MN, USA). Antibody against NF- $\kappa$ B (p65) and Sp3 were obtained from Santa Cruz Biotechnology (Santa Cruz, CA, USA). Antibodies against actin and Spl were purchased from Millipore (Billerica, MA, USA). Antibody against acetyl lysine-histone 3 was purchased from Cell Signaling Technology (Beverly, MA, USA).

Construction of VEGF-C promoter plasmids and the luciferase assay. Genomic DNA was extracted from MCF-7 cells using an extraction kit (Qiagen, Hilden, Germany) and the -1046/+38 region from translational start site of VEGF-C gene(NM_005429) was amplified by PCR using two specific primers (VEGF-C1046-forward 5'-ATACTCGAGCTTTTACAACCCCCAGG ACA-3' and VEGF-C-1046-reverse 5'-AAAAAGCTTAGAGA ACACGCCACAGAGAAG-3'). A 1.1-kb DNA fragment was subcloned into the luciferase reporter gene vector pGL3 (Promega, Madison, WI, USA) by XhoI and HindIII to yield the luciferase reporter construct pGL3-1.1kb-VEGF-C (-1046/+38). Using this construct as a template, two 5'-deletion constructs were generated by the following primers: VEGF-C-439-forward 5'-ATACTCGAGCTCTCACTTCGGGGAAGG-3' and VEGF-C-185-forward 5'-ATACTCGAGGCCCTGCAAAGTT GGGAAC-3'. MDA-MB-231 cells were seeded into 6-well plates and transfected with $1 \mu \mathrm{g}$ of serial VEGF-C promoterluciferase plasmids. After $24 \mathrm{~h}$, cells were incubated without or with SAHA $(10 \mu \mathrm{M})$ for an additional $24 \mathrm{~h}$. The luciferase activity was detected by reporter assay system (Promega),
Table I. PCR primers used in this study.

$\begin{array}{ll}\text { VEGF-C } & \text { F: 5'-CAGTTACGGTCTGTGTCCAGTGTAG-3' } \\ \text { VEGF-C } & \text { R:5'-GGACACACATGGAGGTTTAAAGAAG-3' } \\ \text { Sp1 } & \text { F: 5'-TATAGCAAATGCCCCAGGT-3' } \\ \text { Sp1 } & \text { R: 5'-TTGCCATACACTTTCCCACA-3' } \\ \text { Sp3 } & \text { F: 5'-CCTGCAGATATTAGGATCAAGG-3' } \\ \text { Sp3 } & \text { R: 5'-GCCTCTGTAATTCATCACTTCG-3' } \\ \text { RelA } & \text { F: 5'-TCAATGGCTACACAGGACCA-3' } \\ \text { RelA } & \text { R: 5'-ATCTTGAGCTCGGCAGTGTT-3' } \\ \text { Actin } & \text { F: 5'-TGTTACCAACTGGGACGACA-3' } \\ \text { Actin } & \text { R: 5'-GGGGTGTTGAAGGTCTCAAA-3' }\end{array}$

F, forward; R, reverse.

according to the manufacturer's instructions, and normalized by protein concentration in cell lysates.

Transient cell transfection. GFP-Sp1 expression vector (kindly provided by Dr Jan-Jong Hung, National Cheng Kung University, Taiwan) and control vector were transfected into MDA-MB-231 cells using Lipofectamine 2000 (Invitrogen). After 48 h, cells were treated with SAHA for an additional $24 \mathrm{~h}$. Conditioned medium and cell lysates were harvested for further analysis.

Reverse transcription-polymerase chain reaction (RT-PCR). MDA-MB-231, MCF-7, BT-474 and M10 breast cells were seeded into the 6-well plates. Following overnight incubation, cells were treated with different concentrations of SAHA for $24 \mathrm{~h}$. Total RNA was isolated by RNeasy mini kit (Qiagen) and mRNAs were reverse-transcribed to cDNA by MMLV reverse transcriptase (Promega) using oligo-dT primers according to the manufacturer's instructions. PCR reaction was performed under the following conditions: initialization step for $5 \mathrm{~min}$ at $95^{\circ} \mathrm{C}, 30$ cycles of amplification, with $40 \mathrm{sec}$ at $95^{\circ} \mathrm{C}$ for denaturation and $40 \mathrm{sec}$ at $60^{\circ} \mathrm{C}$ for annealing and $40 \mathrm{sec}$ at $72^{\circ} \mathrm{C}$ for elongation, and $7 \mathrm{~min}$ at $72^{\circ} \mathrm{C}$ for further extension. The PCR primers used are shown in Table I.

Enzyme-linked immunosorbent assay (ELISA) for VEGF-C. MDA-MB-231 cells were incubated under various concentrations of SAHA in L15 medium for $24 \mathrm{~h}$. The conditioned medium was collected and centrifuged at $1500 \mathrm{rpm}$ for $5 \mathrm{~min}$ to remove cell debris. VEGF-C concentration was measured using Quantikines Human VEGF-C Immunoassay kit from R\&D.

Western blotting. Cells were seeded into the 6-well plates. Following overnight incubation, cells were treated with different concentrations of SAHA for 6, 12 or $24 \mathrm{~h}$. Cells were washed with cold PBS and lysed in RIPA buffer $(50 \mathrm{mM}$ Tris- $\mathrm{HCl}, \mathrm{pH} 7.4,50 \mathrm{mM} \mathrm{NaCl}, 1 \mathrm{mM}$ EDTA, 0.5 M sucrose, $0.25 \%$ sodium deoxycholate, $10 \%$ glycerol, $1 \%$ NP-40; protease inhibitors were added prior to use) on ice for $10 \mathrm{~min}$. Cell debris was removed by centrifugation and cellular proteins were further fractionated by SDS-PAGE and transferred onto polyvinylidene difluoride membranes (Millipore). 


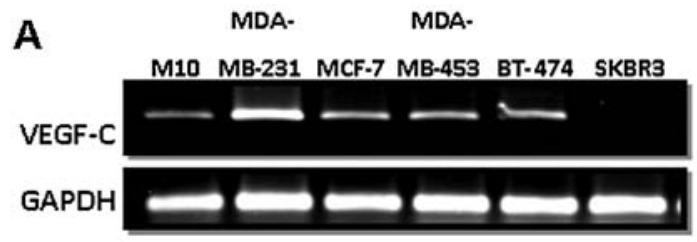

B

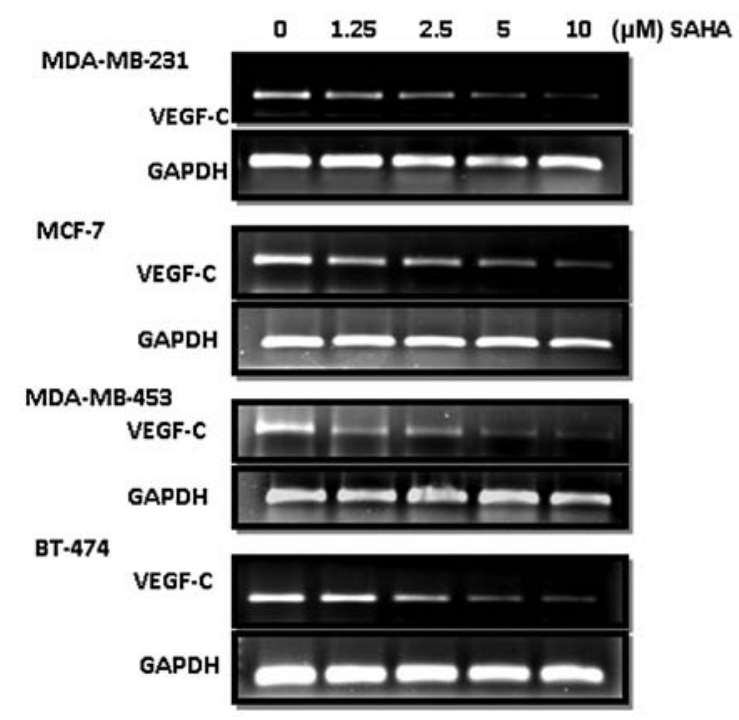

Figure 1. Expression of VEGF-C in breast cancer cell lines and its inhibition by SAHA. (A) VEGF-C expression of various breast cancer cell lines compared to the normal human mammary epithelial cell line M10. (B) Breast cancer cell lines were treated with different concentrations of SAHA for $24 \mathrm{~h}$. VEGF-C mRNA was detected by RT-PCR.

After incubation with 5\% non-fat milk in TBST, the membranes were probed with different primary antibodies followed by horseradish peroxidase-conjugated anti-mouse or anti-rabbit antibodies. Enhanced chemiluminescence (ECL) reagent (Millipore) was used to detect the blots according to the manufacturer's instructions.

Statistical analysis. Data were expressed as the means \pm SE. Student's t-test was used to evaluate the differences between various experimental groups. $\mathrm{p}<0.05$ was considered to indicate a statistically significant difference.

\section{Results}

VEGF-C expression is inhibited by SAHA in breast cancer cell lines. As shown in Fig. 1A, we found that all the cell lines, except SKBR3, investigated in this study expressed higher levels of VEGF-C than the normal breast epithelial cell line M10. SAHA dose-dependently downregulated the expression of VEGF-C in MDA-MB-231, MCF-7, MDA-MB-453 and BT-474 cells (Fig. 1B).

Reduction of VEGF-C production by SAHA. We next investigated whether production of the VEGF-C protein was indeed inhibited. Since MDA-MB-231 cells expressed the highest amount of VEGF-C, we used this cell line as a model in the subsequent experiments. Two approaches were performed. First, cellular VEGF-C protein level was determined by western
A

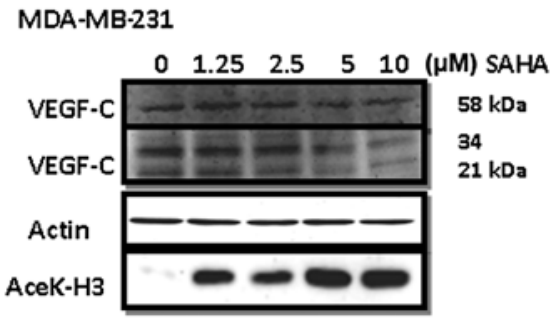

B

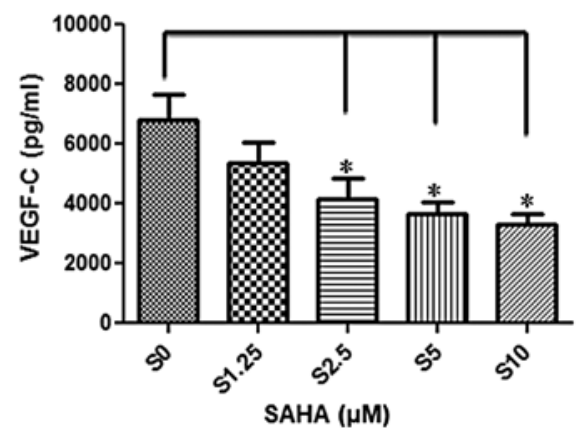

Figure 2. Inhibition of VEGF-C protein expression and production by SAHA. (A) MDA-MB-231 cells were treated with different concentrations of SAHA for $24 \mathrm{~h}$ and cellular proteins and histones were extracted. VEGF-C isoforms with a molecular weight of 58,34 and $21 \mathrm{kDa}$ were detected by western blotting. Acetylation status of histone $\mathrm{H} 3$ was also investigated by anti acetyl-lysine $\mathrm{H} 3$ antibody. (B) MDA-MB-231 cells were incubated under various concentrations of SAHA in L15 medium for $24 \mathrm{~h}$. The conditioned medium was collected and VEGF-C concentration was measured using ELISA assay. ${ }^{*} \mathrm{p}<0.05$, compared to the control group.

blotting. As shown in Fig. 2A, three isoforms of VEGF-C with a molecular weight of 58, 34 and $21 \mathrm{kDa}$ were reduced by SAHA in a dose-dependent manner. We also found that histone $\mathrm{H} 3$ acetylation was significantly increased indicating SAHA at these concentrations indeed exerted potent inhibitory effects on HDACs. In addition, VEGF-C released into the conditioned medium detected by ELISA assay was also reduced accordingly. Treatment of $10 \mu \mathrm{M}$ SAHA reduced VEGF-C concentration by 50\% (Fig. 2B).

SAHA represses VEGF-C promoter activity via the $-185 /+38$ of the promoter region. Since SAHA reduces VEGF-C mRNA levels, this drug might directly inhibit VEGF-C transcription. We cloned human VEGF-C promoter region between $-1046 /+38$ bp region from translational start site and generated different deletion promoter-luciferase constructs (Fig. 3A). These constructs were transfected into MDA-MB-231 and the effect of SAHA was examined. Our data demonstrated that SAHA at the concentration of $10 \mu \mathrm{M}$ inhibited the fulllength $(-1046 /+38)$ promoter by $30-40 \%$ (Fig. 3B). Deletion of promoter to -185 region did not affect SAHA-induced inhibition indicating the responsive elements were located between $-185 /+38$ region.

SAHA reduces Spl-mediated VEGF-C expression. Bioinformatics search revealed several potential transcription factor binding sites including Sp1, AP-2 and NF- $\mathrm{BB}$ within this region. Notably, we found that SAHA caused reduction of Sp1

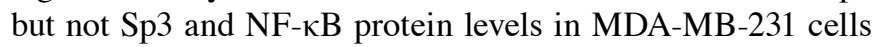


A

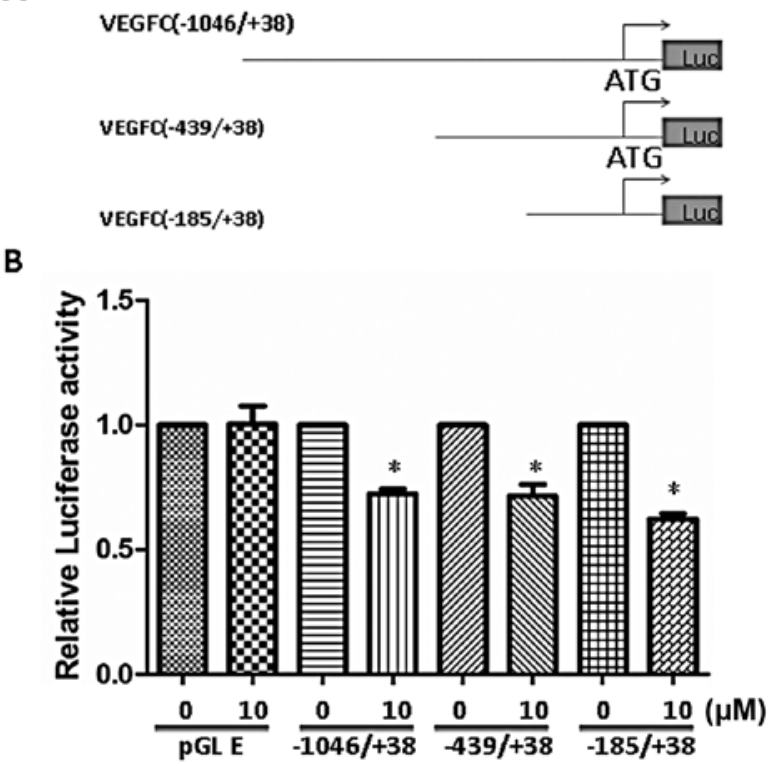

Figure 3. SAHA inhibits human VEGF-C gene promoter activity. (A) Human VEGF-C gene promoter $(-1046 /+38)$ was amplified from MCF-7 cells and subcloned into pGL3 reporter vector. Two additional deletion mutant reporters were generated. (B) MDA-MB-231 cells were seeded into 6-well plates and transfected with $1 \mu \mathrm{g}$ of serial VEGF-C promoter-luciferase plasmids. After $24 \mathrm{~h}$, cells were incubated without or with SAHA $(10 \mu \mathrm{M})$ for an additional $24 \mathrm{~h}$. Luciferase activity was detected by the reporter assay system and normalized by protein concentration in cell lysates. ${ }^{*} \mathrm{p}<0.05$, compared to the control group.

(Fig. 4A). Our data demonstrated that the Spl protein level was significantly decreased at $12 \mathrm{~h}$ after SAHA addition. However, no significant change at the mRNA level of Sp1, Sp3 and NF- $\mathrm{kB}$ was found until a 24-h treatment suggesting SAHA inhibited Sp1 via a post-translational regulation (Fig. 4B). To
A

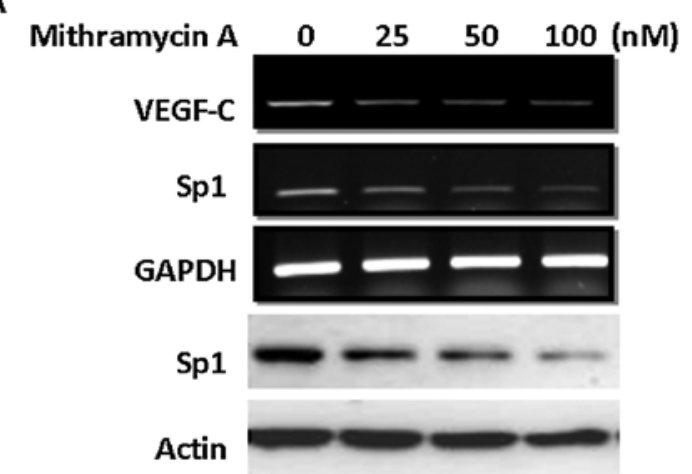

B

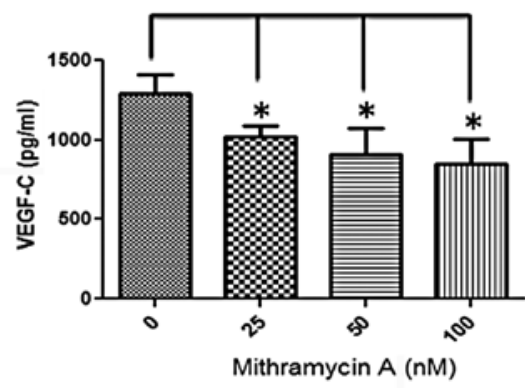

Figure 5. Inhibition of Sp1 reduces VEGF-C expression and production. (A) MDA-MB-231 cells were treated with different concentrations of Sp1 inhibitor mithramycin A for $24 \mathrm{~h}$. Expression of Sp1 and VEGF-C was studied. (B) Conditioned medium was also collected and VEGF-C concentration was examined. ${ }^{\mathrm{p}}<0.05$, compared to the control group.

confirm the importance of Sp1, we treated cells with an Sp1 inhibitor mithramycin A and found that this inhibitor reduced Sp1 protein and attenuated VEGF-C mRNA expression dose-dependently (Fig. 5A). The amount of VEGF-C in the conditioned medium was also significantly reduced (Fig. 5B).

A

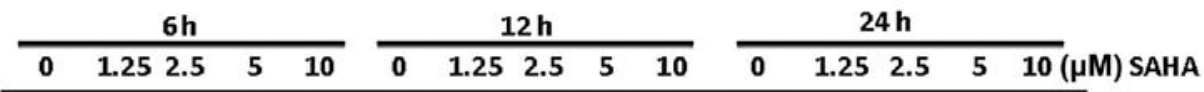

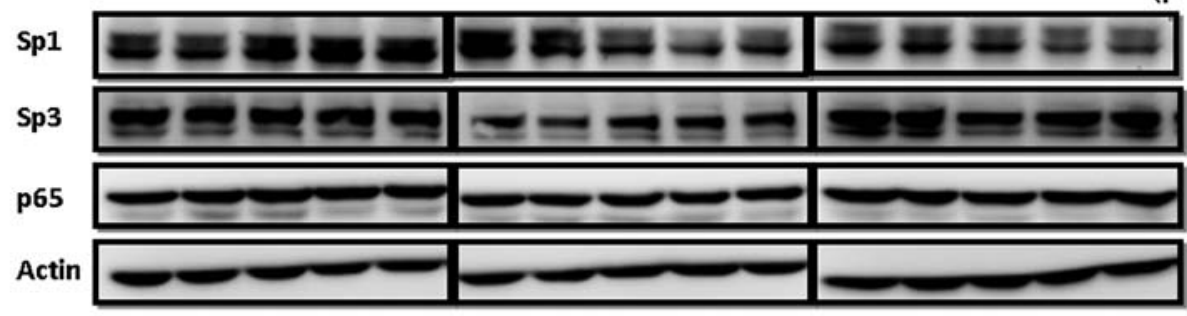

B

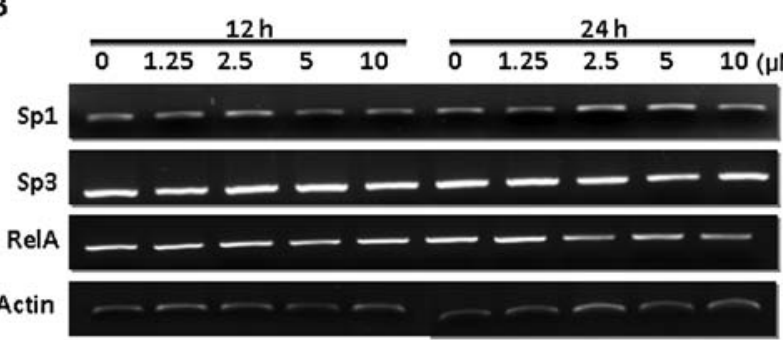

Figure 4. SAHA downregulates Sp1 protein but not mRNA levels. (A) MDA-MB-231 cells were treated with different concentrations of SAHA for 6, 12 or $24 \mathrm{~h}$. Cellular proteins were harvested for western blot analysis. Protein levels of Sp1, Sp3 and p65 were determined. (B) Cellular mRNAs were also harvested at 12 or $24 \mathrm{~h}$ after SAHA treatment and mRNA levels of Sp1, Sp3 and RelA were examined by RT-PCR. 
A

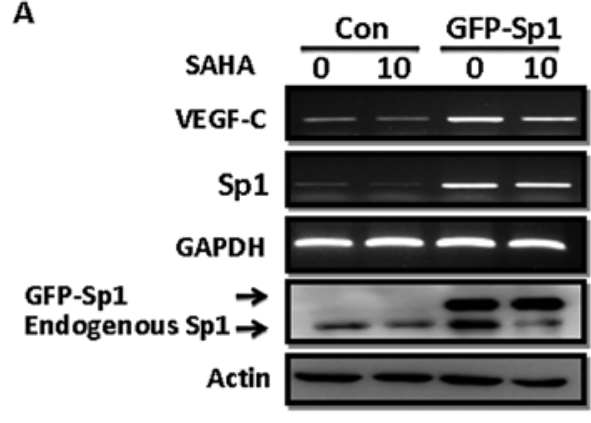

B

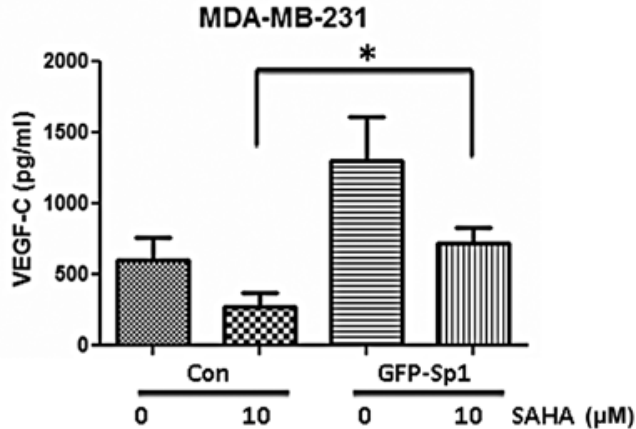

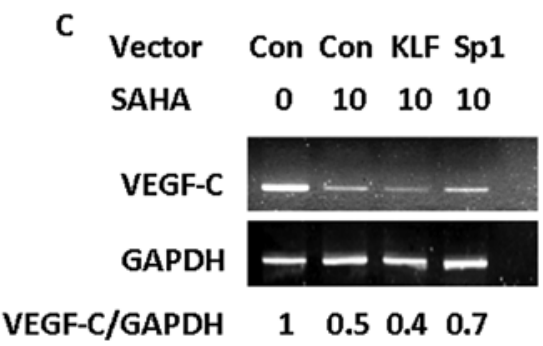

Figure 6. Enforced expression of Sp1 partly reverses the inhibition of VEGF-C by SAHA. (A) MDA-MB-231 cells were transfected with control (Con) or GFP-Sp1 vector for $24 \mathrm{~h}$ and were then treated without or with $10 \mu \mathrm{M}$ of SAHA for another $24 \mathrm{~h}$. Cellular mRNAs and proteins were harvested and expression of VEGF-C and Sp1 was examined. (B) Conditioned medium was also collected and VEGF-C concentration was examined. "p $<0.05$ when the results of two experimental groups were compared. (C) MDA-MB-231 cells were transfected with Con, KLF10 (KLF) or GFP-Sp1 (Sp1) vector for 24 h and then treated without or with $10 \mu \mathrm{M}$ of SAHA for another $24 \mathrm{~h}$. VEGF-C mRNA level was examined by RT-PCR and was normalized to GAPDH. The ratio of the densitometric signal of VEGF-C/GAPDH is indicated.

Enforced expression of Sp1 partially reverses the inhibition of VEGF-C by SAHA. The aforementioned data suggest that SAHA inhibited VEGF-C expression partly via Sp1. Consistent with this hypothesis, enforced expression of Sp1 in MDA-MB231 cells partly reversed the inhibition of VEGF-C by SAHA (Fig. 6A). The VEGF-C released into the medium was also restored by Spl overexpression (Fig. 6B, lanes 2 and 4). Ectopic expression of $\mathrm{Sp1}$ alone increased VEGF-C mRNA expression and the amount of VEGF-C in the conditioned medium. However, this upregulation was still attenuated by SAHA as this drug caused significant reduction of endogenous $\mathrm{Sp} 1$ protein as shown in Fig. 6A. The effect of Sp1 is specific since expression of KLF10, another member of the Kruppel-like/Sp1 gene family, could not antagonize the inhibition of VEGF-C by SAHA (Fig. 6C). Collectively, our results suggest that SAHA induced downregulation of $\mathrm{Sp} 1$ protein via a post-translational mechanism which led to reduction of Sp1-driven VEGF-C expression in breast cancer cells.

\section{Discussion}

Pathological studies have demonstrated that expression of VEGF-C and its receptor VEGFR3 is associated with angiogenesis, lymphangiogenesis and lymph node metastasis in breast cancer $(22,23)$. By using an orthotopic transplantation model, Skobe et al clearly showed that induction of lymphangiogenesis by VEGF-C promotes breast cancer metastasis (24). Therefore, targeting VEGF-C/VEGFR3 signaling axis is important for breast cancer therapy. Since VEGFR3 is a tyrosine kinase, tyrosine kinase inhibitors are potential candidates to inhibit VEGFR3 activity. Sorafenib was initially identified as a potent inhibitor of c-RAF. However, it also inhibits VEGFR2 and VEGFR3 at concentrations of approximately 6-10 nM (25). This drug is now used in the clinic for the treatment of solid tumors. Other kinase inhibitors including sunitinib, AMG706, axitinib, and AZD2171 have also shown inhibitory activity against VEGFR3 and are now under different phases of clinical trials (26). Another strategy to suppress VEGFR3 activity is by blocking antibodies. A recombinant bispecific antibody has been developed to neutralize the biological activities of VEGFR2 and VEGFR3 (27). Roberts et al also demonstrated that inhibition of VEGFR3 activation with the antagonistic antibody potently suppresses lymph node and distant metastasis in an orthotopic spontaneous breast cancer model (28). An antibody that inhibits homodimerization of VEGFR3 and its heterodimerization with VEGFR2 has recently been shown to suppress both angiogenesis and lymphangiogenesis in vivo (29).

The targeting of VEGF-C has not received significant attention in the past decade. By using antibody phage-display, Rinderknecht et al identified a VEGF-C-blocking antibody which could effectively inhibit the interaction between VEGF-C and VEGFR3 and suppress its downstream signaling (30). The underlying mechanism mediating the upregulation of VEGF-C in cancer cells is largely unclear. A pioneer study of the genomic organization of human and mouse VEGF-C genes revealed that the upstream sequences contain conserved putative binding sites for Sp1, AP-2, and NF- $\mathrm{BB}$ transcription factors but not TATA box (31). In the present study, we demonstrated for the first time that a clinically used histone 
deacetylase inhibitor SAHA was able to inhibit Sp1-mediated expression of VEGF-C in human breast cancer cells and reduced VEGF-C concentration in the conditioned medium. Results of this study suggest that multiple transcription factors may be simultaneously involved in the regulation of VEGF-C. In addition, we demonstrated that the Spl specific inhibitor mithramycin A also attenuated VEGF-C expression suggesting a critical role of Sp1 in VEGF-C transcription. Our results provide a new strategy to suppress the expression of a lymphangiogenic factor in cancer cells by using HDAC inhibitors. Reduction of the production of VEGF-C will attenuate lyphangiogenesis and lymphatic metastasis in vivo. Since SAHA has already been approved for cancer treatment in the clinic, it will be of benefit to test whether this drug, in addition to its cytotoxic effect, also shows anti-lymphangiogenic activity in animals and patients.

$\mathrm{Sp} 1$ is a ubiquitous transcription factor expressed in mammalian cells and was initially recognized as a constitutive activator of housekeeping genes. However, recent studies demonstrated that $\mathrm{Spl}$ is involved in the control of tissue-specific and inducible genes and these target genes play critical roles in proliferation, differentiation, apoptosis and oncogenesis (32). In addition, increased $\mathrm{Sp} 1$ protein was found in various types of human cancer suggesting a possible oncogenic role $(33,34)$. Our finding that SAHA causes downregulation of Sp1 protein is noteworthy. Although the underlying mechanism remains unclear, two potential mechanisms may be involved. First, a previous study identified lysine-703 as a major acetylation site of Sp1. Inhibition of HDAC activity by SAHA may directly affect the acetylation status of Sp1 and subsequently change protein stability (35). Second, heat shock protein 90 (hsp90) is an important regulator of Spl stability (36). SAHA has been demonstrated to inhibit the HDAC6-hsp90 chaperone complex to induce the degradation of client proteins such as mutant p53 (37). Therefore, it seems possible that SAHA may inhibit hsp90 and then affect Sp1 degradation.

In the present study, we demonstrated that SAHA is a potent inhibitor of VEGF-C expression in breast cancer cells and we showed that the inhibition is mediated by repression of Sp1. Our results also suggest that SAHA may exert antilymphangiogenic activity in cancer treatment.

\section{Acknowledgements}

This study was supported by grant CA-101-PP-39 from the National Health Research Institutes and DOH 101-TD-C-111-002 and DOH 101-TD-C-111-004 from the Department of Health, Taiwan, R.O.C.

\section{References}

1. Sarkies P and Sale JE: Cellular epigenetic stability and cancer. Trends Genet 28: 118-127, 2012.

2. Esteller $\mathrm{M}$ and Herman JG: Cancer as an epigenetic disease: DNA methylation and chromatin alterations in human tumours. J Pathol 196: 1-7, 2002.

3. Kouzarides T: Chromatin modifications and their function. Cell 128: 693-705, 2007.

4. Huang L and Pardee AB: Suberoylanilide hydroxamic acid as a potential therapeutic agent for human breast cancer treatment. Mol Med 6: 849-866, 2000.

5. Kim MS, Kwon HJ, Lee YM, et al: Histone deacetylases induce angiogenesis by negative regulation of tumor suppressor genes. Nat Med 7: 437-443, 2001.
6. Butler LM, Agus DB, Scher HI, et al: Suberoylanilide hydroxamic acid, an inhibitor of histone deacetylase, suppresses the growth of prostate cancer cells in vitro and in vivo. Cancer Res 60: 5165-5170, 2000.

7. Marks P, Rifkind RA, Richon VM, Breslow R, Miller T and Kelly WK: Histone deacetylases and cancer: causes and therapies. Nat Rev Cancer 1: 194-202, 2001.

8. Minucci S and Pelicci PG: Histone deacetylase inhibitors and the promise of epigenetic (and more) treatments for cancer. Nat Rev Cancer 6: 38-51, 2006.

9. Marks PA and Breslow R: Dimethyl sulfoxide to vorinostat: Development of this histone deacetylase inhibitor as an anticancer drug. Nat Biotechnol 25: 84-90, 2007.

10. Duvic $\mathrm{M}$ and $\mathrm{Vu} \mathrm{J}$ : Vorinostat: a new oral histone deacetylase inhibitor approved for cutaneous T-cell lymphoma. Expert Opin Investig Drugs 16: 1111-1120, 2007.

11. Peart MJ, Tainton KM, Ruefli AA, et al: Novel mechanisms of apoptosis induced by histone deacetylase inhibitors. Cancer Res 63: 4460-4471, 2003.

12. Shao Y, Gao Z, Marks PA and Jiang X: Apoptotic and autophagic cell death induced by histone deacetylase inhibitors. Proc Natl Acad Sci USA 101: 18030-18035, 2004.

13. Gui CY, Ngo L, Xu WS, Richon VM and Marks PA: Histone deacetylase (HDAC) inhibitor activation of p21 ${ }^{\mathrm{WAF} 1}$ involves changes in promoter-associated proteins, including HDAC1. Proc Natl Acad Sci USA 101: 1241-1246, 2004.

14. Mitsiades CS, Mitsiades NS, McMullan CJ, et al: Transcriptional signature of histone deacetylase inhibition in multiple myeloma: Biological and clinical implications. Proc Natl Acad Sci USA 101: 540-545, 2004.

15. Khan AN and Tomasi TB: Histone deacetylase regulation of immune gene expression in tumor cells. Immunol Res 40: 164-178, 2008.

16. Ogbomo H, Michaelis M, Kreuter J, Doerr HW and Cinatl J Jr: Histone deacetylase inhibitors suppress natural killer cell cytolytic activity. FEBS Lett 581: 1317-1322, 2007.

17. Deroanne CF, Bonjean K, Servotte S, et al: Histone deacetylases inhibitors as anti-angiogenic agents altering vascular endothelial growth factor signaling. Oncogene 21: 427-436, 2002.

18. Alitalo K, Tammela T and Petrova TV: Lymphangiogenesis in development and human disease. Nature 438: 946-953, 2005.

19. Mandriota SJ, Jussila L, Jeltsch M, et al: Vascular endothelial growth factor-C-mediated lymphangiogenesis promotes tumour metastasis. EMBO J 20: 672-682, 2001.

20. Maula SM, Luukkaa M, Grenman R, Jackson D, Jalkanen S and Ristamaki R: Intratumoral lymphatics are essential for the metastatic spread and prognosis in squamous cell carcinomas of the head and neck region. Cancer Res 63: 1920-1926, 2003.

21. Dadras SS, Paul T, Bertoncini J, et al: Tumor lymphangiogenesis: a novel prognostic indaicator for cutaneous melanoma metastasis and survival. Am J Pathol 162: 1951-1960, 2003.

22. Valtola R, Salven P, Heikkila P, et al: VEGFR-3 and its ligand VEGF-C are associated with angiogenesis in breast cancer. Am J Pathol 154: 1381-1390, 1999.

23. Wang CA, Jedlicka P, Patrick AN, et al: Six1 induces lymphangiogenesis and metastasis via upregulation of VEGF-C in mouse models of breast cancer. J Clin Invest 122: 1895-1906, 2012.

24. Skobe M, Hawighorst T, Jackson DG, et al: Induction of tumor lymphangiogenesis by VEGF-C promotes breast cancer metastasis. Nat Med 7: 192-198, 2001.

25. Flaherty KT: Sorafenib in renal cell carcinoma. Clin Cancer Res 13: S747-S752, 2007.

26. Hanrahan EO and Heymach JV: Vascular endothelial growth factor receptor tyrosine kinase inhibitors Vandetanib (ZD6474) and AZD2171 in lung cancer. Clin Cancer Res 13: S4617-S4622, 2007.

27. Jimenez X, Lu D, Brennan L, et al: A recombinant, fully human, bispecific antibody neutralizes the biological activities mediated by both vascular endothelial growth factor receptors 2 and 3. Mol Cancer Ther 4: 427-434, 2005.

28. Roberts N, Kloos B, Cassella M, et al: Inhibition of VEGFR-3 activation with the antagonistic antibody more potently suppresses lymph node and distant metastases than inactivation of VEGFR-2. Cancer Res 66: 2650-2657, 2006.

29. McDonald DM: New antibody to stop tumor angiogenesis and lymphatic spread by blocking receptor partnering. Cancer Cell 18: 541-543, 2010.

30. Rinderknecht M, Villa A, Ballmer-Hofer K, Neri D and Detmar M: Phage-derived fully human monoclonal antibody fragments to human vascular endothelial growth factor-C block its interaction with VEGF receptor-2 and 3. PLoS One 5: e11941, 2010. 
31. Chilov D, Kukk E, Taira S, et al: Genomic organization of human and mouse genes for vascular endothelial growth factor C. J Biol Chem 272: 25176-25183, 1997.

32. Li L and Davie JR: The role of Sp1 and Sp3 in normal and cancer cell biology. Ann Anat 192: 275-283, 2010.

33. Wang L, Wei D, Huang S, et al: Transcription factor Sp1 expression is a significant predictor of survival in human gastric cancer. Clin Cancer Res 9: 6371-6380, 2003.

34. Safe S and Abdelrahim M: Sp transcription factor family and its role in cancer. Eur J Cancer 41: 2438-2448, 2005.
35. Hung JJ, Wang YT and Chang WC: Sp1 deacetylation induced by phorbol ester recruits p300 to activate 12(S)-lipoxygenase gene transcription. Mol Cell Biol 26: 1770-1785, 2006.

36. Wang SA, Chuang JY, Yeh SH, et al: Heat shock protein 90 is important for Sp1 stability during mitosis. J Mol Biol 387 1106-1119, 2009.

37. Li D, Marchenko ND and Moll UM: SAHA shows preferential cytotoxicity in mutant p53 cancer cells by destabilizing mutant p53 through inhibition of the HDAC6- Hsp90 chaperone axis. Cell Death Differ 19: 1268-1276, 2012. 